\title{
Salud bucal colectiva en Colombia: un campo en construcción ${ }^{*}$
}

\author{
Oral Health Research in Colombia: a field under construction
}

Pesquisa de Saúde Bucal na Colômbia: Um campo em construção

\author{
Carolina Morales Borrero', Lorena Maldonado Maldonado². \\ 1 PhD Salud Pública, magíster en Administración en Salud, odontóloga, Universidad Nacional de Colombia, Colombia. mcmoralesb@ \\ unal.edu.co, carolinamorabo@yahoo.es ORCID: https://orcid.org/0000-0001-6824-5395 \\ 2 Magíster en Salud Pública, odontóloga, Universidad Nacional de Colombia, Colombia. lamaldonadom@unal.edu.co, ORCID: https:// \\ orcid.org/0000-0002-0997-9804
}

Recibido: 03/10/2018. Aceptado: 06/12/2019. Publicado: 20/03/2020

Morales Borrero C, Maldonado Maldonado L. Salud bucal colectiva en Colombia: un campo en construcción. Rev. Fac. Nac. Salud Pública. 2020; e335716. DOI: https://doi.org/10.17533/udea.rfnsp.e335716

\section{Resumen}

La salud bucal constituye un componente esencial de la salud general, por las funciones y los procesos vitales que sustenta y que hacen posible las dimensiones materiales y simbólicas de la vida humana. En el contexto latinoamericano, el abordaje de la salud bucal como un asunto social y complejo, que trasciende la odontología biomédica convencional, se denomina "salud bucal colectiva" y ha tenido distintos desarrollos en los países, siendo Brasil su máximo exponente. De esta manera, este texto busca caracterizar el proceso de construcción del campo social de la salud bucal colectiva en Colombia. Para ello, se hace una revisión de literatura publicada en bases de datos especializadas entre los años 2005 y 2016, período en que se presentaron avances en la formulación de políticas de salud bucal globales, regionales, nacionales y locales, con el fin de interpretar las publicaciones en salud bucal en Colombia, a la luz de los desarrollos que ha tenido Brasil, identificando los avances y desafíos del país en este campo. En síntesis, se observa que el campo social de la salud bucal colectiva en Colombia está en construcción, requiere esfuerzos en investigación, prácticas interdisciplinarias y movilización social, en diálogo con otras corrientes de pensamiento crítico en salud.

-Palabras clave: Salud bucal colectiva; salud pública; salud colectiva; odontología.

* Este ensayo es resultado de la investigación "Construcción de capacidades de investigación en atención primaria en salud y salud oral”, una de las realizadas en el marco del Semillero de Investigación en Salud Colectiva, el cual hace parte de la convocatoria del Programa Nacional de Semilleros de Investigación, Creación e Innovación de la Universidad Nacional de Colombia 2013-2015, de la Dirección de Investigación de Sede (DIB) de la misma institución [1]. 


\begin{abstract}
Oral health constitutes an essential component of general health, due to the vital functions and processes that it supports and that make possible the material and symbolic dimensions of human life. In the Latin American context, the approach to oral health as a social and complex issue, which transcends conventional biomedical dentistry, is called "collective oral health" and has had different developments in the countries, with Brazil being its greatest exponent. In this way, this text seeks to characterize the process of construction of the social field of collective oral health in Colombia. For this, a review of literature published in specialized databases between 2005 and 2016 is carried out, a period in which progress was made
\end{abstract}

in the formulation of global, regional, national and local oral health policies, in order to interpret the publications on oral health in Colombia, in light of the developments that Brazil has had, identifying the progress and challenges of the country in this field. In summary, it is observed that the social field of collective oral health in Colombia is under construction, it requires research efforts, interdisciplinary practices and social mobilization, in dialogue with other currents of critical thinking in health.

-Key words: Collective oral health; public health; collective health; odontology.

\section{Resumo}

A saúde bucal constitui um componente essencial da saúde geral, devido às funções e processos vitais que apóia e que possibilitam as dimensões material e simbólica da vida humana. No contexto latino-americano, a abordagem da saúde bucal como uma questão social e complexa, que transcende a odontologia biomédica convencional, é chamada de "saúde bucal coletiva" e teve desenvolvimentos diferentes nos países, sendo o Brasil o seu maior expoente. Dessa forma, este texto busca caracterizar o processo de construção do campo social da saúde bucal coletiva na Colômbia. Para isso, é realizada uma revisão da literatura publicada em bancos de dados especializados entre 2005 e 2016, período em que houve progresso na formulação de políticas globais, regionais, nacionais e locais de saúde bucal, a fim de interpretar os publicações sobre saúde bucal na Colômbia, à luz dos desenvolvimentos que o Brasil teve, identificando os avanços e desafios do país nesse campo. Em resumo, observase que o campo social da saúde bucal coletiva na Colômbia está em construção, requer esforços de pesquisa, práticas interdisciplinares e mobilização social, em diálogo com outras correntes de pensamento crítico em saúde.

---------Palavras-Chave: Saúde bucal coletiva; saúde pública; saúde coletiva; odontologia

\section{Introducción}

Las perspectivas sobre el fenómeno de la salud bucal (SB) que se producen en este siglo han cambiado: se tratan nuevos temas y han surgido teorías sociales desde propuestas interdisciplinarias que sustentan integralmente dicho fenómeno, contemplando las funciones vitales, los componentes y las dimensiones que se reúnen en lo bucal. Actualmente, se reconoce la importancia de la SB para el bienestar humano, el buen vivir y el ejercicio del derecho a la salud [2], ya que la boca sustenta funciones vitales como la alimentación, la respiración, la comunicación, la expresión, el afecto, la sexualidad y la interacción entre los seres humanos, y de estos con la naturaleza [3]; además, sus componentes subjetivos y materiales, con expresión en lo orgánico, biológico, cultural, político y social, contribuyen a delinear la singularidad de la identidad humana y las diferencias entre grupos sociales y comunidades dentro de los contextos que le dan existencia $[4,5]$.

Tradicionalmente, la SB se ha estudiado dentro del modelo biomédico mediante el positivismo metodológico o filosofía positiva, que afirma que el único conocimiento auténtico es aquel derivado del método científico empleado por las ciencias físiconaturales modernas; de esta forma, se ha asumido un monismo metodológico que incurre en reducciones de aquellas ciencias y del conocimiento que se producen sobre fenómenos sociales y humanos complejos como la SB [8]. Desde la perspectiva biomédica tradicional, la SB no se concibe como un fenómeno complejo; por el contrario, para dicho enfoque, se reduce a aspectos orgánicos y biológicos individuales de la cavidad bucal y a elementos asistenciales-clínicos de la práctica odontológica enmarcada en las orientaciones que surgen de la medicalización de la salud [6]. En

El concepto de campo es tomado del sociólogo francés Pierre Bourdieu, quien lo define como un espacio social de acción en el que permanentemente convergen influencias, fuerzas y redes sociales, algunas veces en conflicto. Estas influencias se definen en su existencia y en la relación con las demás. Pensar la salud como un campo implica entenderla en términos de relaciones. Dependiendo de las reglas que regulen 
contraste, este trabajo explora la SB como un campo de conocimiento y acción, ${ }^{\dagger}$ en donde se reconoce la complejidad de este fenómeno.

Como una alternativa al reduccionismo biomédico tradicional, se desarrolla la salud colectiva latinoamericana ( $\mathrm{SCL}$ ), impulsada por comunidades científicas en Brasil, México, Ecuador y Argentina, que conforma un campo social dedicado al estudio de las relaciones entre las condiciones de vida, los modelos económicos y las políticas para el buen vivir de las sociedades, cuestionando el positivismo en salud, las formas de medicalización y las políticas de salud que buscan unificar e imponer parámetros hegemónicos del modelo biomédico global. Así mismo, han incorporado, de forma crítica, en el estudio de los componentes y las dimensiones de la salud, diversas ciencias y disciplinas como las ciencias sociales, la antropología médica, la economía política, entre otras, y han visibilizado la necesidad de los trabajos interdisciplinarios en el abordaje de la salud [10].

En el marco de la SCL, se desarrolla el campo de la salud bucal colectiva ( $\mathrm{SBC}$ ), que busca ampliar y complejizar las perspectivas sobre la SB como parte constitutiva de la vida de los seres humanos, identificando relaciones entre las dimensiones individual, colectiva y social de este fenómeno. En lo individual, las expresiones biológicas genotípica y fenotípica del individuo no se encuentran aisladas en la bucalidad de cada quien, sino atada a la dimensión colectiva y social de la SB, porque es en el cuerpo en donde se materializan todos aquellos procesos sociales y comunitarios, que ocurren en tiempos y en espacios concretos. En lo colectivo, se observan las expresiones particulares de los grupos sociales, de la forma de vivir, trabajar y agenciar una mejor salud. Estas expresiones están ligadas a los territorios específicos donde se construye y se guarda la memoria de colectiva de la SB [11]. En ese sentido, la SBC explica la bucalidad en sus dimensiones individuales, colectivas y sociales, mediante esquemas interdisciplinares y comunitarios que reconocen la singularidad y la diversidad del proceso salud-enfermedad bucal como parte de una totalidad social $[11,12]$.

La SBC no se limita a un quehacer de la academia, de las instituciones estatales o de las políticas públicas; también incorpora a las comunidades en la identificación, el análisis y la resolución de problemáticas y necesidades de SB [13]. Al estar enmarcada en el campo de la SCL, la SBC comparte con esta la perspectiva multidimensional y transectorial que, como lo expresaba Silva Paim, "abarca un conjunto articulado de prácticas técnicas, ideológicas, políticas y económicas desarrolladas en el ámbito académico, en las organizaciones de salud y en las instituciones de investigación vinculadas a distintas corrientes de pensamiento resultantes de proyectos de reforma en el campo de la salud" [14, p. 158].

Brasil, país pionero y líder en la creación y la construcción del campo de la SBC, ha tenido importantes desarrollos en este campo desde los años ochenta, con la reforma al sistema social de la salud [15], basado en propuestas de las ciencias sociales y humanas críticas que reconocen el derecho a la salud y la SB, y la necesidad de intervenir las inequidades que se producen y se reproducen en un modelo civilizatorio, excluyente y capitalista [16]. El abordaje de la SB desde el marco epistemológico de la SCL en Brasil, le ha permitido a este país avanzar en una comprensión más amplia de los problemas y las necesidades de las comunidades en materia de SB y, así mismo, generar nuevas formas de responder a dichas necesidades desde la $\mathrm{SBC}[17,18]$.

En Colombia, la SBC se ha desarrollado de forma distinta a la de Brasil, debido a diferentes trayectorias en los procesos sociales de reivindicación del derecho a la salud y a la SB, y a las diferencias en la configuración de los sistemas de salud: mientras en Brasil se logra establecer un sistema sanitario universalista, en Colombia se instaura un mercado regulado de servicios de salud desde una lógica neoliberal, con graves consecuencias en la garantía efectiva del derecho a la salud [19].

Todo lo anterior motiva este ensayo, cuyo objetivo es caracterizar el proceso de construcción del campo social de la SBC en Colombia. Para ello, a partir de una revisión de la literatura y tomando como referente a Brasil, se identifican avances y desafíos de la SBC en Colombia, y se visibilizan las tendencias y los temas de interés de las comunidades académicas, y los actores que investigan y difunden conocimiento al respecto. En últimas, con ello se busca aportar al campo de la SBC, tanto en lo local como en lo regional.

las relaciones en un campo, se define su estructura y el carácter dominante o no de sus partes y del campo propiamente tal. La salud como campo comprende la acción de agentes tales como las comunidades profesionales o académicas, con sus vínculos nacionales e internacionales y sus tradiciones; las comunidades y pacientes, con sus problemas y necesidades en salud, pero también con capacidades de organización y transformación; las agremiaciones y expresiones organizativas; los agentes del Estado y del mercado, quienes intervienen en mayor o menor medida, en la configuración de las profesiones, del profesional de la salud y del campo [7].

* La SCL es una corriente de pensamiento crítico en salud que surge como respuesta a la crisis de la salud pública convencional en los años setenta, con expresiones de conocimiento y formación no hegemónica en salud en México; de sistemas universales de salud en Brasil; de diálogo entre la salud colectiva, ambiente y sociedad en Ecuador, y del conflicto social y político con la salud pública en Colombia [9] 


\section{La salud bucal colectiva brasileña}

En Brasil, la corriente de la SBC se inicia desde los años sesenta, con la odontología sanitaria basada en la obra de Mario Cháves, quien impulsó una desmercantilización de los servicios odontológicos, con el fin de atender las necesidades de las comunidades, particularmente de aquellas más vulneradas [20]. A partir de este desarrollo, se impulsan diferentes prácticas en $\mathrm{SB}$, con énfasis en acciones preventivas y comunitarias, que permiten una atención más integral a las comunidades, configurándose así lo que hoy se denomina SBC. Esta corriente toma fuerza a finales de los años ochenta, en el estado de São Paulo, bajo la influencia del Movimiento de Salud Colectiva y de la Asociación Brasilera de Salud Bucal Colectiva (Abrasbuco) en el marco del Sistema Único de Salud (sus) [21,22].

La SBC de Brasil se configura como un campo científico y político, que articula conocimientos, métodos de trabajo interdisciplinarios y prácticas odontológicas, que trascienden la atención individual y el énfasis técnico-clínico, propio de la odontología mercantil [23]. Así mismo, desarrolla la bucalidad como un concepto que motiva la acción en favor de las funciones sociales de la boca, y desmonopoliza y recupera la SB de las manos del mercado [20,24]. Esta SBC se constituye en un eje orientador esencial de sus para el logro de la universalidad, la equidad y la garantía del derecho fundamental a la SB; de allí que la Tercera Conferencia Nacional de SBC, realizada en Brasilia entre el 29 de julio al 1 de agosto de 2004, con participación de 883 delegados, ${ }^{*}$ integra la SBC en la agenda pública como un indicador de inclusión social y responsabilidad de todos los actores de la sociedad [25].

El estudio de la SB desde el marco epistémico de la SBC lleva a profundizar las dimensiones colectivas y sociales del sus, las condiciones y los modos de vivir de las comunidades, y los determinantes sociales de la salud de los individuos y sus familias [21]. En este sentido, se reconoce el carácter social e histórico del proceso salud-enfermedad bucal y el compromiso ético y político de la odontología con el buen vivir de las comunidades $[10,13,14]$. Estas dimensiones que aborda la SBC obligan a redefinir el sujeto/objeto de estudio de la odontología, y a orientar las prácticas de los equipos de salud, para participar social y políticamente en los proyectos comunitarios dirigidos a mejorar la SB de las comunidades $[6,17]$.

Lo anterior hace que la SBC brasilera asuma una posición frente al Estado, la sociedad y al mercado. No se restringe a producir servicios estatales de odontología

* Los delegados representan a usuarios de servicios de salud, trabajadores de la salud y gestores de servicios, seleccionados en conferencias municipales y regionales. de carácter filantrópico o acciones comunitarias de carácter caritativo-asistencial, los cuales son generados para personas pobres vistas como sujetos sin capacidad de agenciar su SB en el mercado. La SBC no busca crear jerarquías en los servicios de odontología de acuerdo con el uso de tecnologías o conocimientos específicamente innovadores, porque se opone a reproducir nociones de colonialidad entre países de la región. Esta forma de ver la SB propende por acciones individuales y comunitarias en todas las dimensiones existentes de la SB. Se cuestiona el fenómeno de mercantilización de la SB, al redefinir su objeto de estudio en el marco del campo de la salud colectiva, cuyo compromiso histórico y social con la ciudadanía es superar el sentido acumulativo del capital o el autoritarismo de Estado, para favorecer el derecho a la SB y el buen vivir.

Este derecho rompe con el statu quo de la mercantilización de los servicios y del monopolio de acceso a recursos por parte de las élites. Busca acceso universal a servicios de promoción de la salud, prevención y atención bajo principios éticos; cuidados integrales con acceso a todos los niveles de complejidad en la atención y, control popular del sistema de servicios de SB dentro de la seguridad y protección social [24].

\section{La salud bucal colectiva colombiana a la luz de los avances de Brasil}

Al caracterizar la producción y la difusión científica colombiana sobre la SB durante 12 años consecutivos, se puede observar y describir el proceso de construcción del campo de la SBC en Colombia. Los investigadores y las investigadoras colombianas han desarrollado trabajos multi e interdisciplinarios que acercan comunidades académicas distintas, al estudiar problemas de la SB, con la existencia de un interés creciente por esta, más allá de la ciencia moderna convencional. La introducción de interrogantes sobre los determinantes sociales de la salud, sobre las desigualdades y la determinación social de la inequidad, así como sobre los perfiles epidemiológicos de la SB de la población atrae al campo enfoques críticos de diversas ciencias y experiencias comunitarias [26]; asimismo, surgen alternativas de manejo de las problemáticas referidas a la SB, como también respuestas a las crisis que atraviesa la profesión odontológica, cuestionada por su baja capacidad resolutiva y por su escaso impacto social [27-29].

Para caracterizar el proceso de construcción del campo social de la SBC en Colombia, se realiza una búsqueda de literatura publicada en las bases de datos especializadas SciencieDirect, Medline, EBSCO, Lilacs, SciELO y Latindex, entre los años 2005 y 2016; se toma este período, debido a que durante estos años se formulan e implementan varias políticas de carácter global, 
regional, nacional y local que inciden sobre LA SB. En la esfera global, se inicia la primera década del siglo XXI con la formulación, por parte de las Naciones Unidas, de los "Objetivos de desarrollo del Milenio" [30]; la Organización Mundial de la Salud da a conocer el reporte de la Comisión sobre los determinantes sociales de la salud [31] y también el reporte mundial de SB con metas muy específicas [32]. A nivel regional, en Brasil se privilegia el derecho a la SB como un derecho esencial, en la declaración de la carta de Brasilia [33]. En Colombia, en el ámbito nacional, se implementan políticas y reformas al sistema de salud con efectos sobre la SB, como el Plan Decenal de Salud Pública [34] y la definición de los mecanismos nacionales y locales para la concreción de un Plan Nacional de Salud Bucal, mediante la Resolución 3577 de 2006 [35]; y en lo local, se formulan las primeras políticas de SB en tres importantes ciudades del país, las cuales ocurren, en orden cronológico, en Bogotá (2004-2010) [36], Manizales (2007-2017) [37] y más recientemente en Medellín (2013-2022) [38].

Como criterios de inclusión para la revisión de la literatura se establecieron: documentos con texto completo disponible; alusión directa a la SB (con los términos "Salud bucal", "Salud colectiva", "Salud bucal colectiva") o alusión indirecta a la SB, con temas, enfoques, métodos de investigación y prácticas relacionadas con dichos términos. Se selecciona un total de 77 artículos, que cumplen los criterios de inclusión.

\section{Comportamiento general de la salud bucal colectiva de Colombia}

Acerca del abordaje de la SB, se observan nuevas tendencias y viejos énfasis. Dentro de los asuntos de estudio preferidos, la enfermedad sigue siendo uno de los más comunes y especialmente la caries. Otras patologías que adquieren interés por las dinámicas epidemiológicas a nivel nacional son: fluorosis, enfermedad periodontal, cáncer oral, maloclusiones y anomalías craneofaciales.

También se incursiona en el estudio de la SB con perspectivas y temas que cuestionan los enfoques preventivistas y las políticas con énfasis gerencial, entre ellos, el estudio de los determinantes sociales de la SB, la equidad, el enfoque de derechos, el análisis crítico de las políticas en SB, de los servicios de SB y la atención primaria en salud (APS).

Asimismo, aparecen otros temas novedosos, referidos a la formación de los profesionales en odontología y su rol en la SB colectiva o pública, la salud ocupacional como aspecto importante en la garantía del derecho a la SB, y la iatrogenia en odontología como obstáculo del sistema de salud al logro del derecho a la SB.

Las representaciones sociales de la SB y la calidad de vida son temas que se siguen estudiando, aunque desde enfoques similares y con poco cambio.

En la figura 1 se muestran los porcentajes de aparición, en las publicaciones en Colombia entre 2005 y 2016, de las temáticas sobre SB abordadas.

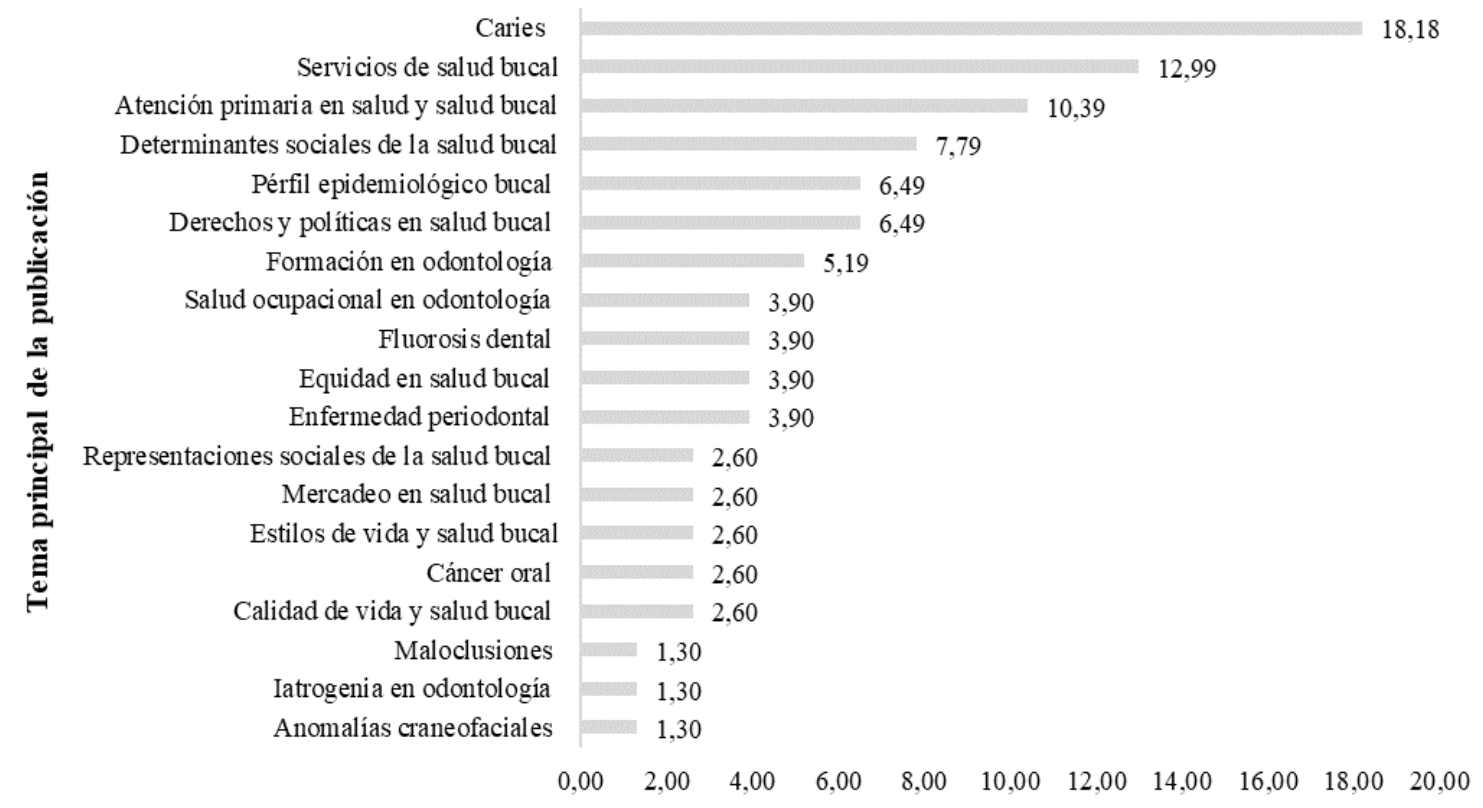

Figura 1. Porcentajes de aparición de las temáticas sobre salud bucal abordadas en las publicaciones en Colombia, 2005-2016 
Entre los autores de las publicaciones, se destacan los investigadores de instituciones públicas, con el 75,3\% del total de publicaciones $(n=58)$; los de instituciones privadas, en un $17 \%(n=13)$; y en menor medida, los investigadores de alianzas público-privadas, cuyos trabajos son el 7,8 \% $(n=6)$ del total de publicaciones.

Respecto a los grupos poblacionales más estudiados, se destacan los niños, generalmente de comunidades escolares (menores de 12 años), seguidos de los profesionales de la salud, los estudiantes de las facultades de odontología y poblaciones vulneradas en general.
Las poblaciones que más se estudian se ubican en territorios urbanos y ciudades capitales de departamentos, con universidades de larga tradición en investigación. En orden decreciente, las poblaciones estudiadas están en Antioquia, Bolívar, Valle del Cauca, Nariño y Bogotá (véase figura 2). Llama la atención la escasez de estudios en territorios rurales, debido posiblemente a las dificultades existentes para superar las condiciones de vida marginalizada, incluso para acceder a recursos para investigar.

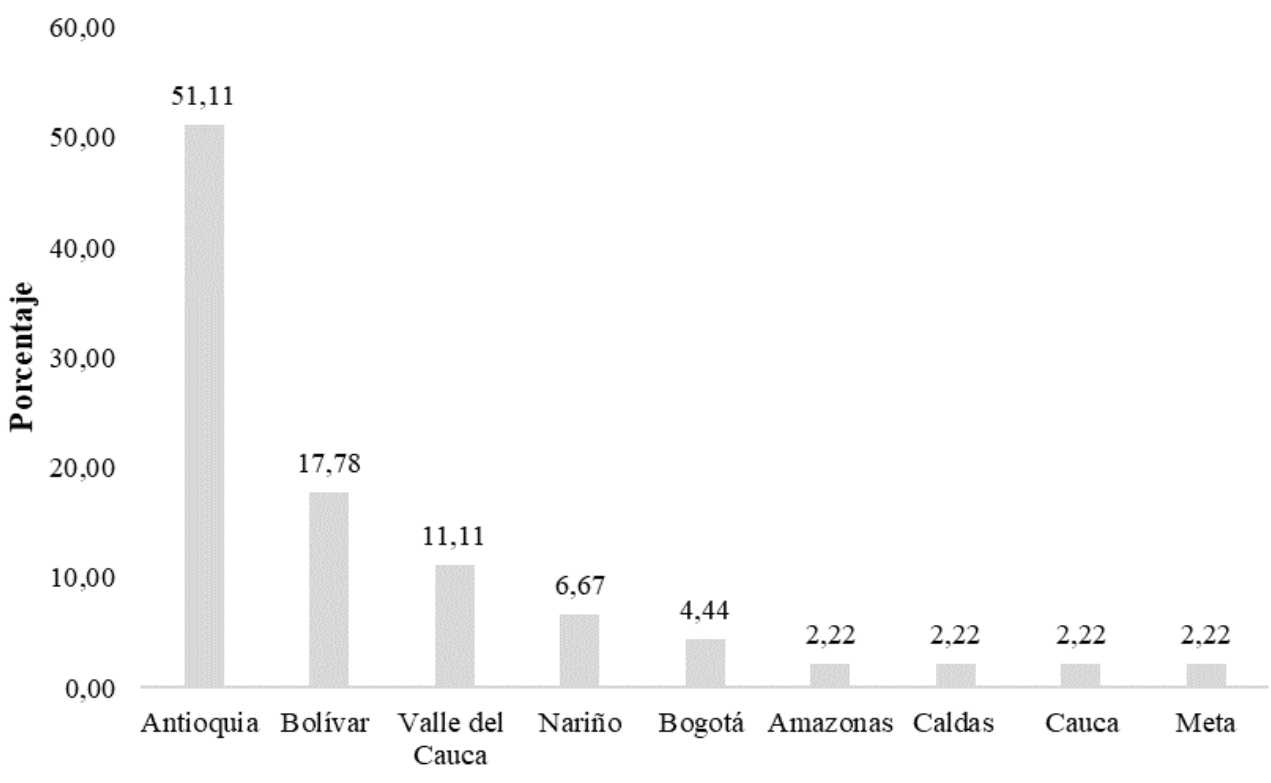

Ubicación geográfica

Figura 2. Localización geográfica de las poblaciones estudiadas, 2005-2016

En cuanto a la difusión del conocimiento producido, el idioma español es el preferido para las publicaciones, en $87,0 \%(n=67)$ del total de artículos. En inglés se publica el 6,5\% $(n=5)$, y en inglés y español, el 6,5\% $(n=5)$. Las revistas seleccionadas por los investigadores para la publicación son, principalmente, de circulación nacional $(88,3 \%)$.

Según el área de conocimiento, las revistas donde más se aceptan las publicaciones sobre los temas observados son de odontología y salud pública y ciencias políticas, seguidas por revistas de medicina (véase tabla 1).

En general, la mayoría de las revistas donde se publica pertenecen a universidades del país, dentro de las cuales se destacan las revistas CES Odontología de la Universidad ces y la Revista Facultad de Odontología
Tabla 1. Área de conocimiento de las revistas donde se publica sobre los temas observados

\begin{tabular}{lll}
\hline \multicolumn{1}{c}{ Área de conocimiento } & $\mathrm{n}$ & \multicolumn{1}{c}{$\%$} \\
\hline Odontología & 31 & 40,26 \\
\hline Salud pública y ciencias políticas & 31 & 40,26 \\
\hline Medicina & 10 & 12,99 \\
\hline Ciencias económicas & 2 & 2,59 \\
\hline Bioética & 1 & 1,30 \\
\hline Ciencias básicas & 1 & 1,30 \\
\hline Enfermería & 1 & 1,30 \\
\hline Total & 77 & 100,00 \\
\hline
\end{tabular}


Universidad de Antioquia, de Medellín, seguidas por la Revista de Salud Pública de la Universidad Nacional de Bogotá, la Revista Facultad Nacional de Salud Pública de la Universidad de Antioquia de Medellín, y la Revista Gerencia y Políticas de Salud, de la Universidad Javeriana de Bogotá. Las publicaciones internaciones se realizan principalmente en revistas españolas (véase tabla 2).
Respecto de la cantidad de producción y difusión científica en el periodo estudiado, existe una tendencia a aumentar entre los años 2008 y 2014, periodo de amplia discusión, en Colombia, sobre el derecho a la salud y los determinantes sociales de la salud, y de mayor visibilización de la SCL en la academia nacional (Figura 3).

Tabla 2. Revistas con artículos publicados sobre salud bucal colectiva en Colombia y en el extranjero. 2005-2016

\begin{tabular}{|c|c|c|c|}
\hline País & Nombre de revista & Institución & $\begin{array}{l}\text { Número de } \\
\text { publicaciones }\end{array}$ \\
\hline \multirow{16}{*}{ Colombia } & CES Odontología & Universidad CES & 12 \\
\hline & $\begin{array}{l}\text { Revista Facultad de Odontología } \\
\text { Universidad de Antioquia }\end{array}$ & Universidad de Antioquia & 12 \\
\hline & Revista de Salud Pública & Universidad Nacional de Colombia & 9 \\
\hline & $\begin{array}{l}\text { Revista Facultad Nacional de Salud } \\
\text { Pública }\end{array}$ & Universidad de Antioquia & 8 \\
\hline & Revista Gerencia y Políticas de Salud & Pontificia Universidad Javeriana & 8 \\
\hline & Colombia Médica & Universidad del Valle & 5 \\
\hline & Acta Odontológica Colombiana & Universidad Nacional de Colombia & 3 \\
\hline & Universitas Odontológica & Pontificia Universidad Javeriana & 3 \\
\hline & Biomédica & $\begin{array}{l}\text { Instituto Nacional de Salud de } \\
\text { Colombia }\end{array}$ & 1 \\
\hline & Biosalud & Universidad de Caldas & 1 \\
\hline & $\begin{array}{l}\text { Investigación y Educación en } \\
\text { Enfermería }\end{array}$ & Universidad de Antioquia & 1 \\
\hline & Revista Colombiana de Bioética & Universidad del Bosque & 1 \\
\hline & Revista de la Facultad de Medicina & Universidad Nacional de Colombia & 1 \\
\hline & $\begin{array}{l}\text { Revista Escuela de Administración y } \\
\text { Negocios }\end{array}$ & Universidad EAN & 1 \\
\hline & Hacia la Promoción de la Salud & Universidad de Caldas & 1 \\
\hline & Salud Uninorte & Universidad del Norte & 1 \\
\hline España & Revista Clínica de Medicina de Familia & $\begin{array}{l}\text { Sociedad Castellano-Manchega } \\
\text { de Medicina de Familia y } \\
\text { Comunitaria } 3\end{array}$ & 3 \\
\hline Brasil & Ciência \& Saúde Coletiva & $\begin{array}{l}\text { Asociación Brasilera de Salud } \\
\text { Colectiva }\end{array}$ & 1 \\
\hline Dinamarca & European Journal of Dental Education & $\begin{array}{l}\text { Association for Dental Education in } \\
\text { Europe (ADEE) }\end{array}$ & 1 \\
\hline Chile & $\begin{array}{l}\text { International Journal of } \\
\text { Odontostomatology }\end{array}$ & Universidad de la Frontera & 1 \\
\hline Estados Unidos & $\begin{array}{l}\text { Journal of Immigrant and Minority } \\
\text { Health }\end{array}$ & $\begin{array}{l}\text { Case Western Reserve University } \\
\text { School of Medicine }\end{array}$ & 1 \\
\hline Cuba & Revista Cubana de Salud Pública & $\begin{array}{l}\text { Centro Nacional de Información } \\
\text { de Ciencias Médicas }\end{array}$ & 1 \\
\hline México & Salud Pública de México & $\begin{array}{l}\text { Instituto Nacional de Salud Pública } \\
\text { de México }\end{array}$ & 1 \\
\hline Total & & & 77 \\
\hline
\end{tabular}




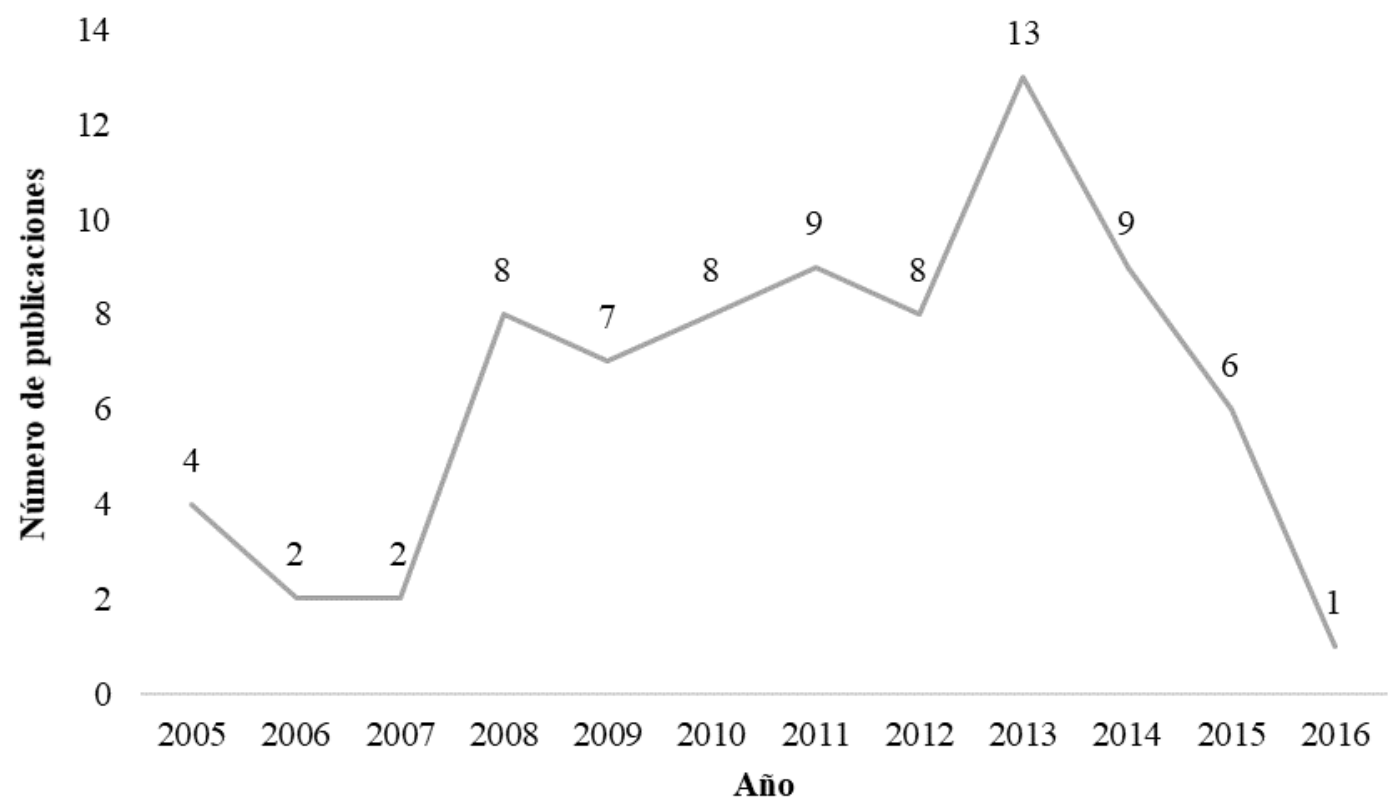

Figura 3. Cantidad de publicaciones sobre salud bucal colectiva en Colombia por año. 2005-2016

\section{Avances y desafíos del campo de la salud bucal colectiva en Colombia}

A la luz de la experiencia de Brasil, se puede afirmar que la SBC colombiana es un campo que está en construcción. Hemos pasado de una etapa inicial dedicada a concurrir en escenarios informales para la discusión del proceso salud-enfermedad-atención, con escasa investigación o difusión de resultados de los estudios, a otra etapa de formalización de perspectivas, discursos, prácticas y formas organizadas de construir y difundir conocimientos sobre la SBC.

En esta segunda etapa, las escuelas e instituciones de formación de pre y posgrados en las facultades de odontología, así como las investigaciones y actividades de las comunidades académicas de Medellín, Cali, Bogotá, Bucaramanga y Manizales, han aportado y propiciado espacios de debate, como mecanismo de ruptura de los paradigmas más convencionales de la odontología.

La creciente difusión de los temas novedosos se puede ubicar en una tercera etapa en el desarrollo de la SBC en Colombia, consistente en la diferenciación entre la producción y la difusión de conocimiento, con fortalecimiento de la difusión desde la segunda década de este siglo. Al igual que en Brasil, se favorece la investigación ligada a las instituciones de prestación de servicios y de formulación de políticas, y se crean observatorios (Observatorio Nacional de la Salud Bucal y la Práctica odontológica de la Facultad de Odontología de la Universidad de Antioquia, 2006; Observatorio para la equidad en calidad de vida y salud, de la Secretaría de Salud de Bogotá, 2009) para hacer seguimiento a los resultados de las acciones políticas en SB.

Contrario a Brasil, en Colombia, la profundización de la privatización de la salud y la seguridad social limitan la universalidad del derecho a la SB. Los estudios publicados por la Universidad de Antioquia avanzan en explicar este problema y proponen reorientar la SB, las políticas y los actores del sistema de salud hacia las propuestas de la SBC [39].

El campo de SBC cambia las perspectivas de análisis sobre la SB. En Colombia, este cambio es más visible entre los años estudiados: 2005 y 2016. En ese periodo, se pasa de un esquema de análisis dicotómico de la SB, con énfasis en la enfermedad, a un abordaje desde perspectivas más complejas, con esquemas que entienden la enfermedad a partir de teorías biologicistas, ecologicistas, naturalistas y de factores de riesgo, y la superan, profundizando, además, en las explicaciones de los procesos sociohistóricos que determinan la inequidad social y sanitaria, y su impacto diferencial en la SB. Ya en ese período se emplean fundamentos y categorías derivadas de la tradición del materialismo histórico, de la economía, de la antropología y de las ciencias políticas, utilizadas en la SCL, para aproximarse a la dimensión social de la SB. La noción de salud bucal como fenómeno social se amplía para incluir dimensiones sociales y 
colectivas que abren posibilidades para pensarla como un derecho fundamental y para cuestionar la odontología convencional, aproximándose a la propuesta de SBC desarrollada en Brasil.

En el desarrollo de la SBC en Colombia se ha fomentado paulatinamente el interés por temas como la APS, la promoción de la salud, la prevención de la enfermedad, el acceso oportuno y de calidad a servicios sanitarios y la equidad en SB. La APS se estudia también desde perspectivas que buscan superar las limitaciones del sistema de salud vigente en Colombia y su modelo de atención. Se proponen manejos intersectoriales y trabajo en equipos de APS para poblaciones vulnerables y vulneradas, y se incorporan significados de la SB para responder con más precisión a las necesidades y particularidades de género, clase, etnia y ciclo vital $[5,10,40]$.

Los desarrollos en SBC en Colombia, aunque menores en relación con los avances en Brasil dada su relación con el sus-, han logrado permear algunos escenarios importantes de la política pública nacional, destacándose el último estudio nacional de SB (ENSAB IV), que se realiza bajo el marco teórico de la epidemiología crítica y la determinación social de la salud, proveniente de la SCL [41]. Este estudio reconoce los determinantes sociales y las inequidades como núcleos temáticos de la SB y de las políticas, para lograr un abordaje integral en SB.

Por lo anterior, se concluye que el campo social en SBC en Colombia se está construyendo mediante investigaciones y acciones que confrontan los enfoques y los métodos predominantes con las propuestas novedosas no hegemónicas, con un propósito: mejorar el buen vivir, mediante una SBC activa y propositiva. Este campo tiene espacios de formación específica, con esquemas interdisciplinarios, transdisciplinarios y multiprofesionales, donde las comunidades académicas participan y difunden sus avances.

Se recomienda trabajar con otras ciencias y perspectivas de carácter crítico, tales como los estudios feministas y las ciencias de la salud colectiva, mediante estudios multicéntricos que permitan profundizar y visibilizar la importancia de la SB como un derecho fundamental, garantizado por el Estado, de interés colectivo y con participación social. También se requieren nuevos esfuerzos y recursos para realizar estudios sobre la SB en el campo de la SBC, así como investigaciones históricas con abordajes comparativos sobre el campo de la SBC en América Latina, para identificar tendencias, escuelas y desafíos de importancia para el campo. Así mismo, se recomienda insistir en una transformación de la educación odontológica, que permita superar el morbicentrismo tradicional y avanzar hacia una odontología holística y crítica, articulada con las demás ciencias y profesiones de la salud y de la vida.

\section{Agradecimientos}

Agradecemos a la Red Latinoamericana de Salud Bucal Colectiva por estimular la difusión del tema en la región.

\section{Conflicto de interés}

Las autoras declaramos no tener ningún conflicto de interés (real o potencial) con personas o instituciones relacionadas con los resultados presentados en este trabajo.

\section{Financiación}

La Universidad Nacional de Colombia y el Departamento de Salud Colectiva de la misma universidad apoyaron y facilitaron la realización del estudio con recursos de la Sede Bogotá, en el marco del proyecto Programa Nacional de Semilleros de Investigación, Creación e Innovación de la Universidad Nacional de Colombia 2013-2015.

\section{Declaración de responsabilidad}

Declaramos que las afirmaciones y puntos de vista expresados en el cuerpo del documento reflejan exclusivamente la postura de las autoras y no de la institución a la que se encuentran vinculadas.

\section{Declaración de autoría}

Las autoras declaramos que cumplimos con los criterios establecidos por el Comité Internacional de Directores de Revistas Biomédicas (ICMJE), para ser consideradas autoras del presente manuscrito. Cada autora realizó aportes sustanciales en el diseño del ensayo, la revisión, la sistematización y el análisis de información, y en la construcción y el ajuste del documento final. Así mismo, cada autora está en la capacidad y la disposición de responder por el contenido del ensayo y su proceso de elaboración.

\section{Referencias}

1. Piragauta Ruiz MA, Wiches Niño BA. Recuento histórico de un escenario de investigación y acción. En: Morales Borrero C, Maldonado Maldonado LA, editores. Investigación en salud colectiva: entre ciencias sociales y ciencias de la salud. Bogotá: Universidad Nacional de Colombia; 2018. pp. 85-92.

2. Martínez Abreu J, Gispert Abreu E de los Á, Castell-Florit Serrate P, et al. Consideraciones sobre el concepto de salud y su enfoque en relación a la salud bucal. Rev. Méd. Electrón. [internet]. 2014 [citado 2018 nov. 16]; 36(5):671-80. Disponible 
en: http://scielo.sld.cu/scielo.php?script=sci_arttext\&pid=S1684$18242014000500015 \& \operatorname{lng}=$ es\&nrm=iso\&tlng=es

3. Federación Dental Internacional. El desafío de las enfermedades bucodentales. Una llamada a la acción global. Atlas de Salud Bucodental. 2.a ed. Ginebra: Federación Dental Internacional [internet]; 2015 [citado 2019 feb. 15]. Disponible en: https:// www.fdiworlddental.org/sites/default/files/media/documents/ book_spreads_oh2_spanish.pdf.

4. Martínez Delgado CM, López Palacio AM, Londoño Marín BH, et al. Exploración de significados con respecto a la salud bucal de un grupo de gestantes de la ciudad de Medellín, Colombia: hay alfabetización en salud bucal. Rev. Fac. Odontol. Univ. Antioq. [internet]. 2011 [citado 2019 ene. 20]; 23(1):76-91. Disponible en: http://www.scielo. org.co/scielo.php?script=sci arttext\&pid=S0121-246X2011000200 006\&lng $=$ en\&nrm $=$ iso\&tlng $=$ es.

5. Beltrán Salazar M. De lo oral a lo bucal en la odontología. Rev. Colomb. Investig. Odontol. [internet]. 2011 [citado 2019 feb. 11]; 2(5):123-33. Disponible en: https://es.scribd.com/ document/244834497/De-Lo-Oral-a-Lo-Bucal-Magda-BeltranSalazar.

6. Laserna ME. Más allá de la disciplina odontológica... la Salud Bucal Colectiva [internet]. s. f. [citado 2019 ene. 24]; 1-4. Disponible en: https://www.medellin.gov.co/irj/go/km/docs/ wpccontent/Sites/Subporta1\%20de1\%20Ciudadano/Salud/ Secciones/Programas\%20y\%20Proyectos/Documentos/2013/ Salud\%20Bucal/M\%C3\%B3dulo\%20Educaci\%C3\%B3n/La\%20 salud\%20bucal\%20colectiva.pdf

7. Bourdieu P. Campo de poder, campo intelectual [internet]. s. c.: Montressor; 2002 [citado 2020 ene. 26]. Disponible en: http:// www.redmovimientos.mx/2016/wp-content/uploads/2016/10/ Bourdieu-P.-2002.-Campo-de-poder-campo-intelectual.Itinerario-de-un-concepto.-Editorial-Montressor.pdf

8. Quevedo E. El proceso salud-enfermedad: hacia una clínica y una epidemiología no positivistas. En: Salud y administración. Bogotá: Zeus Asesores Ltda; 1992.

9. Morales Borrero C, Rocha Buelvas A. Debate entre salud colectiva y salud pública. En: Morales Borrero C, editor. Salud colectiva y salud pública ¿Se está hablando de lo mismo? [internet]. Bogotá: Universidad Nacional de Colombia; 2017. pp. 31-44. Disponible en: http://www.doctoradosaludp.unal.edu.co/wp-content/ uploads/2018/04/Cuaderno-18.pdf

10. Agudelo Suárez AA, Martínez Herrera E. La salud bucal colectiva y el contexto colombiano: un análisis crítico. Rev. Gerenc. Polit. Salud [internet]. 2009 [citado 2019 mar. 8]; 8(16):91105. Disponible en: http://www.scielo.org.co/pdf/rgps/v8n16/ v8n16a06.pdf.

11. Breilh J. Epidemiología crítica latinoamericana: raíces, desarrollos recientes y ruptura metodológica. En: Morales C, Eslava JC, editores. Tras las huellas de la determinación. Memorias del Seminario Interuniversitario de Determinación Social de la Salud. Bogotá; 2015. pp. 19-76.

12. Laurell AC. La salud-enfermedad como proceso social. Cuad. Méd. Soc. [internet]. 1982 [citado 2019 abr. 12]; 19:1-11. Disponible en: http://capacitasalud.com/biblioteca/wp-content/ uploads/2016/02/Cuadernos-Medico-Sociales-19.pdf.

13. Silva Paim J, Almeida Filho N. Crise da saúde pública e a utopía da saúde coletiva. Salvador, Brazil: Casa da Qualidade Editora; 2000.

14. Silva Paim J. La salud colectiva y los desafíos de la práctica [internet]. s. f. [citado 2019 may. 18]; 151-60. Disponible en: https://repositorio.ufba.br/ri/bitstream/ri/6169/1/Paim JS. La Salud Colectiva. 1992.pdf.
15. Warmling CM, Celeste RK. 4a Reunião de Pesquisa em Saúde Bucal Coletiva: A produção científica nos 10 anos do Brasil sorridente. Rev Fac Odontol [internet]. 2013 [citado 2019 may. 27]; 54:46-65. Disponible en: lume.ufrgs.br/handle/10183/134037.

16. Celeste RK, Warmling CM. Produção bibliográfica brasileira da Saúde Bucal Coletiva em periódicos da saúde coletiva e da odontologia. Ciênc. Saúde Coletiva [internet]. 2014 [citado 2019 jun. 22]; 19(6):1921-32. Disponible en: http:// www.scielo.br/scielo.php?script=sci_arttext\&pid $=$ S141381232014000601921\&lng=pt\&tlng=pt; DOI: http://dx.doi. org/10.1590/1413-81232014196.04932013

17. Narvai PC, Frazão P, Roncalli AG, et al. Cárie dentária no Brasil: declínio, polarização, iniqüidade e exclusão social. Rev Panam Salud Publica/Pan Am J Public Heal [internet]. 2006 [citado 2019 jun. 26]; 19(6):385-393. Disponible en: https://scielosp.org/pdf/ rpsp/2006.v19n6/385-393/pt

18. Nunes ED. Paradigmas de la salud colectiva: breve reflexión. Salud Colect. [internet]. 2014 [citado 2019 jul. 12]; 10(1):57-65. Disponible en: https://scielosp.org/pdf/scol/2014.v10n1/57-65/es

19. Álvarez MH. El derecho a la salud en Colombia: obstáculos estructurales para su realización. Rev. Salud Pública [internet]. 2010 [citado 2019 ago. 24]; 2(2):121-44. Disponible en: http:// www.revistas.unal.edu.co/index.php/revsaludpublica/article/ view/18882\%5Cnhttp://www.revistas.unal.edu.co/index.php/ revsaludpublica/article/download/18882/19840\%5Cnhttp:// www.revistas.unal.edu.co/index.php/revsaludpublica/article/ view/18882/19840.

20. Narvai PC. Saúde bucal coletiva: caminhos da odontologia sanitária à bucalidade. Rev Saude Publica [internet]. 2006 [citado 2019 jun. 20]; 40(N Esp):141-7. Disponible en: http://www. scielo.br/pdf/rsp/v40nspe/30633

21. Botazzo C. Sobre a bucalidade: notas para a pesquisa e contribuição ao debate. Cienc e Saude Coletiva [internet]. 2006 [citado 2019 jul. 17]; 11(1):7-17. Disponible en: http://www. scielo.br/pdf/csc/v11n1/29438.pdf

22. Frazão P. Epidemiologia da oclusão dentária na infância e os sistemas de saúde. Universidade de São Paulo. Tesis [internet]. 1999 [citado 2019 jul. 18]. Disponible en: https://www.teses. usp.br/teses/disponiveis/6/6135/tde-14072016-163502/publico/ DR_415_Frazao_1999.pdf

23. Narvai PC. Saúde bucal coletiva, bucalidade e antropofagia. Ciênc. Saúde Coletiva [internet]. 2006 mar. [citado 2019 abr. 27]; 11(1):18-21. Disponible en: http://www.scielo.br/scielo. php?script $=$ sci_arttext\&pid $=$ S1413-81232006000100003\&ln $\mathrm{g}=$ pt\&tlng=pt; $\quad$ DOI: $\quad$ http://dx.doi.org/10.1590/S141381232006000100003 .

24. Narvai PC, Frazão P. Saúde bucal no Brasil: muito além do céu da boca. Rio de Janeiro: Editora Fiocruz; 2008.

25. Ministério da Saúde, Conselho Nacional de Saúde. 3.a Conferencia Nacional de Saúde Bucal: acesso e qualidade superando exclusão social. Brasília [internet]; 2005 [citado 2020 ene. 20]. Disponible en: http://bvsms.saude.gov.br/bvs/publicacoes/3_conferencia nacional_saude_bucal_relatorio_final.pdf

26. Celeste RK, Broadbent JM, Moyses SJ. Half-century of dental public health research: Bibliometric analysis of world scientific trends. Community Dent Oral Epidemiol [internet]. 2016 [citado 2019 sep. 22]; 44(6):557-63. DOI: https://doi.org/10.1111/ cdoe. 12249

27. Otálvaro Castro G, Salazar S. Transformaciones de la práctica odontológica en el contexto de la reforma estructural del Estado de finales del siglo XX: Confluencias hacia la proletarización de la odontología en Colombia. Rev. Fac. Odontol. Univ. Antioq. 
[internet]. 2014 [citado 2019 jun. 20]; 25:S27-38. Disponible en: https://www.researchgate.net/publication/315115894 Transformaciones_de_la_practica_odontologica_en_el_ contexto_de_la_reforma_estructural_del_Estado_de_finales_ del_siglo_XX_Confluencias_hacia_la_proletarizacion_de_la odontologia_en_Colombia_CHANGES_OF_

28. Jaramillo Delgado G, Velásquez Escobar JP, Marín Uribe A. La odontología: profesión liberal que se debate entre la distinción y la extinción. Rev. Fac. Odontol. Univ. Antioq. [internet]; 2014 [citado 2019 jul. 18]; 25(Supl.):S5-26. Disponible en: http://bibliotecadigital.udea.edu.co/bitstream/10495/5593/1/ JaramilloGonzalo_2014_OdontologiaProfesionLiberal.pdf

29. Franco ÁM, Ochoa EM. La salud bucal colectiva en Medellín: lo que sabemos; lo que desconocemos; lo que queremos y lo normativo [internet]. s. f. [citado 2019 sep. 25]. Disponible en: https:/www.medellin.gov.co/irj/go/km/docs/wpccontent/ Sites/Subporta1\%20de1\%20Ciudadano/Salud/Secciones/ Programas\%20y\%20Proyectos/Documentos/2013/Salud\%20 Bucal/Presentaci\%C3\%B3n\%20SALUD\%20COLECTIVA.ppsx

30. Naciones Unidas. Objetivos de desarrollo del Milenio. Informe 2010 [internet]. 2010 [citado 2020 ene. 24]. Disponible en: https:// web.archive.org/web/20120218061300/http:/www.un.org/es/ comun $/$ docs $/$ ?path $=\% 2$ Fspanish $\% 2$ Fmillenniumgoals $\% 2$ Fpdf $\% 2$ FMDG_Report_2010_SP.pdf.

31. Comisión sobre Determinantes Sociales en Salud. Subsanar las desigualdades en una generación. Alcanzar la equidad sanitaria actuando sobre los determinantes sociales de la salud [internet]. Organización Mundial de la Salud. 2009. Disponible en: http:// www.who.int/social_determinants/thecommission/finalreport/es/.

32. Petersen PE. The World Oral Health Report 2003. Continuous improvement of oral health in the 21st century - the approach of the WHO Global Oral Health Programme [internet]. 2003 [citado 2019 jul. 28]. Disponible en: https://www.who.int/oral_health/ media/en/orh_report03_en.pdf

33. Coordinadores Nacionales de Salud Bucal Latino-Americanos. Carta de Brasilia sobre Salud Bucal en las Américas [internet]; 2009 [citado 2019 sep. 27]. Disponible en: http://189.28.128.100/dab/docs/ publicacoes/geral/carta_brasilia_salud_bucal_americas.pdf
34. Colombia, Ministerio de Salud y Protección Social. Plan Decenal de Salud Pública 2012-2021 [internet]. 2013. Disponible en: https://www.minsalud.gov.co/Documentos y Publicaciones/Plan Decenal - Documento en consulta para aprobación.pdf

35. Colombia, Ministerio de la Protección Social. Resolución 3577, por la cual se adopta el Plan Nacional de Salud Bucal (2006 sep. 28).

36. Secretaría Distrital de Salud. Política de salud oral de Bogotá, D. C. con participación social para el decenio 2011-2021 [internet]. 2011 [citado 2020 mar. 14]. Disponible en: http://www. saludcapital.gov.co/Polit_sectoriales/Salud_oral.pdf

37. Alcaldía de Manizales, Secretaría de Salud Pública, Comité de Salud Oral. Política de salud oral Manizales 2007 / 2017. Manizales; 2007.

38. Secretaría de Salud de Medellín, Universidad de Antioquia. Lineamientos de política pública de salud bucal de Medellín 2013-2022 [internet]. 2015 [citado 2019 ago. 20]. Disponible en: http://www.udea.edu.co/wps/wcm/connect/udea/6f16d8c0-aa8949f5-8d35-948c1d041c8c/Lineamientos.pdf?MOD=AJPERES

39. Otálvaro Castro GJ, Zuluaga Salazar SM, Jaramillo Delgado G, et al. Políticas de salud bucal en Colombia. Tendencias y puntos críticos para la garantía del derecho a la salud. Univ Odontol [internet]. 2019 [citado 2020 ene. 18]; 38(80):47. Disponible en: https://revistas.javeriana.edu.co/index.php/revUnivOdontologica/ article/view/25634; DOI: https://doi.org/10.11144/Javeriana. uo38-80.psbc.

40. Rueda Martínez G, Albuquerque A. La salud bucal como derecho humano y bien ético. Rev Latinoam Bioética [internet]. 2017 [citado 2019 sep. 21]; 17(32-1):36-59. Disponible en: http://www. scielo.org.co/pdf/rlb/v17n1/v17n1a03.pdf; DOI: http://dx.doi. org/10.18359/rlbi.2299.

41. Colombia, Ministerio de Salud y Protección Social. IV Estudio Nacional de Salud Bucal - ENSAB IV [internet]. Bogotá D.C.; 2015 [citado 2019 jul. 20]. Disponible en: https://www.minsalud. gov.co/sites/rid/Lists/BibliotecaDigital/RIDE/VS/PP/ENSABIV-Situacion-Bucal-Actual.pdf 